\title{
Supervivencia de cirugía de rescate en carcinoma escamoso de orofaringe
}

\section{Survival outcomes of salvage surgery in oropharyngeal squamous cell carcinoma}

\author{
Mario Tapia C'ㄹ, Felipe Cardemil $\mathbf{M}^{2,3}$.
}

\begin{abstract}
RESUMEN
La recurrencia de carcinoma de células escamosas orofaríngeo (CCEOF) se asocia a mal pronóstico, particularmente en recurrencias en etapa avanzada. La cirugía en el contexto de rescate es más complicada por el tratamiento oncológico del tumor primario, por lo tanto, tiene un mayor riesgo de complicaciones y estadía hospitalaria. Sin embargo, la cirugía de rescate es la mejor oportunidad del paciente como tratamiento curativo y para supervivencia a largo plazo. La población de pacientes que reciben tratamiento para CCEOF ha cambiado en la última década, se ha reconocido que la incidencia de virus papiloma humano (VPH) asociado a CCEOF ha generado el gran aumento de CCEOF y el cambio asociado en las características de la población de pacientes, ahora los pacientes son más jóvenes y tienen menos comorbilidades. Con el aumento exponencial en la incidencia de CCEOF, la necesidad de cirugía de rescate en CCEOF podría verse en aumento. En vista del aumento de la incidencia de casos con carcinoma escamoso de orofaringe y su importante relación con el VPH, esta revisión se enfoca en la supervivencia tras cirugía de rescate con intención curativa y evaluar si con los avances en su tratamiento ha mejorado su pronóstico.
\end{abstract}

Palabras clave: Neoplasias orofaríngeas, carcinoma de células escamosas, cirugía de rescate, cáncer recurrente de cabeza y cuello, neoplasias de laringe.

\begin{abstract}
Recurrence of oropharyngeal squamous cell carcinoma (OPSCC) is associated with poor prognosis, particularly in advanced stage recurrences. Salvage surgery is complicated by previous oncological treatment of the primary tumor, therefore, it has a higher risk of complications and hospital stay. However, salvage surgery is the patient's best opportunity as a curative treatment and for long-term survival. The population of
\end{abstract}

\footnotetext{
Servicio de Otorrinolaringología, Complejo Asistencial Dr. Víctor Ríos Ruiz, Los Ángeles, Chile.

2 Departamento de Oncología Básico-Clínica y Departamento de Otorrinolaringología, Facultad de Medicina, Universidad de Chile - Hospital San Juan de Dios, Santiago, Chile.

3 Departamento de Otorrinolaringología, Clínica Las Condes, Santiago, Chile.
}

Los autores declaran no tener conflicto de interés.

Recibido el 14 de octubre de 2019. Aceptado el 11 de diciembre de 2019. 
patients receiving treatment for OPSCC has changed in the last decade, it has been recognized that the incidence of human papilloma virus (HPV) associated OPSCC has generated an increase of OPSCC and changes in the epidemiology of the patient population, with younger patients and with less comorbidities. With the exponential increase in the incidence of OPSCC, the need for salvage surgery in OPSCC could increase in the future. In view of the increase in the incidence of cases with squamous oropharyngeal carcinoma and its relationship with HPV, this review focuses on survival after salvage surgery with curative intent and assessing whether the progress in its treatment has improved its prognosis.

Key words: Oropharyngeal neoplasms, squamous cell carcinoma, salvage surgery, recurrent head and neck cancer, larynx neoplasms.

\section{INTRODUCCIÓN}

El cáncer de cabeza y cuello se diagnostica en todo el mundo en aproximadamente 550.000 pacientes y está asociado con aproximadamente 79.000 muertes anuales ${ }^{1}$. El desarrollo y creciente incidencia de carcinoma escamoso de orofaringe (CCEOF) se ha asociado fuertemente con el virus papiloma humano (VPH) ${ }^{2,3}$. Los estudios han mostrado que la presencia de VPH es un factor pronóstico, con generalmente mejor supervivencia en pacientes con CCEOF asociado a VPH, que suelen ser más jóvenes y no fumadores ${ }^{4}$. Sin embargo, las recurrencias después del tratamiento para CCEOF ocurren a pesar de los avances en las modalidades de tratamiento ${ }^{5}$. La supervivencia a largo plazo de pacientes que desarrollan recurrencias se ha reportado desde $23 \%$ a $35 \%$, 6 .

\section{Tratamiento}

El tratamiento del cáncer recurrente de cabeza y cuello representa un gran desafío para todos los miembros del equipo multidisciplinario oncológico de cabeza y cuello. Una mayoría significativa de pacientes con cáncer de laringe y orofaringe en cohortes modernas con estadio temprano 0 avanzado reciben modalidades de tratamiento de preservación de órganos, es decir tratamiento no quirúrgico ${ }^{8-11}$. A pesar de ser éste un tratamiento efectivo, la incidencia de falla local en laringe se ha informado entre $19 \%$ y $33 \%{ }^{12}$. En orofaringe, se ha reportado la incidencia de falla local de $10,2 \%{ }^{13}$ por un centro único europeo y $11,8 \%$ por un gran centro en los Estados Unidos ${ }^{14}$. Por lo tanto, el manejo de la enfermedad recurrente en este contexto generalmente se trata de casos que ya han sido tratados con radioterapia o quimiorradioterapia ${ }^{15}$. Por lo tanto, la cirugía de rescate es habitualmente el tratamiento considerado para estos casos. Sin embargo, la cirugía de rescate extensa se asocia con mayores riesgos de morbilidad y mortalidad ${ }^{16}$.

La recurrencia en cáncer de cabeza y cuello se asocia a mal pronóstico, particularmente en recurrencias en etapa avanzada ${ }^{16}$ e incluso peor si no es tratada ${ }^{17}$. Entre las alternativas de tratamiento se incluye cirugía de rescate, reirradiación, cuidados paliativos 0 enrolamiento en ensayos clínicos, actualmente con estrategias de inmunooncología. La cirugía, en el contexto de rescate, es más complicada por el tratamiento oncológico previo. La calidad del tejido después la radioterapia habitualmente es mala y en consecuencia afecta la cicatrización, un patrón multifocal de recurrencia en un campo irradiado hace la resección desafiante $y$ en general, la salud del paciente luego de su tratamiento previo, con frecuencia está comprometida. La cirugía de rescate, por lo tanto, tiene un mayor riesgo de complicaciones, mayor tiempo de estadía hospitalaria y complicaciones a largo plazo en relación a la deglución y en la competencia de la vía aérea ${ }^{18}$. Sin embargo, la cirugía de rescate es la mejor oportunidad del paciente como tratamiento curativo y para lograr una mayor supervivencia a largo plazo $^{16}$.

Históricamente, el uso de cirugía de rescate en cohortes de pacientes con CCEOF ha sido menor en comparación con casos recurrentes de carcinoma escamoso de laringe (CEL). En una serie de 170 pacientes con CCEOF inicialmente tratados con quimiorradioterapia, 31,4\% (11/35) de las recurrencias recibieron cirugía de rescate ${ }^{19}$. 
En una serie más grande con 1.681 pacientes con CCEOF hubo 199 pacientes con tumores con recurrencia local y $20,6 \%$ de ellos recibieron cirugía de rescate ${ }^{14}$. La población de pacientes que reciben tratamiento para CCEOF ha cambiado en la última década. Se ha reconocido que la aparición de VPH asociada CCEOF ha generado un aumento en la incidencia CCEOF y el cambio asociado en las características de la población de pacientes, siendo ahora los pacientes más jóvenes y con menos comorbilidades $^{20}$. Con el aumento exponencial en la incidencia de $\mathrm{CCEOF}^{2}$, la necesidad de cirugía de rescate en CCEOF podría ir en aumento.

\section{Falla de tratamiento}

Las recurrencias locorregionales son las formas principales de fracaso después del tratamiento primario de un CCEOF. A pesar de los avances en las modalidades de tratamiento, la cirugía sigue siendo la principal forma de tratamiento de rescate para mejorar la supervivencia del paciente en el contexto de la recurrencia. Goodwin y cols. publicaron un metaanálisis de la supervivencia después de la cirugía de rescate para carcinoma escamoso recurrente del tracto aerodigestivo superior ${ }^{16}$ que reportó tasas de supervivencia a 2 y 5 años de $24,6 \%$ y $26 \%$ respectivamente, para pacientes con CCEOF. Hay y cols reportaron que en casos de recurrencias orofaríngeas, $60 \%$ (15/25) se presentaron en un estadio más avanzado que el estadio del tumor primario. La etapa del cuello en el momento de la recurrencia no mostró diferencia entre los casos de laringe y orofaringe con 41 $(67,2 \%)$ de pacientes con cuello N0, $15(24,1 \%)$ con cuello N1 y $5(8,2 \%)$ con cuello N ${ }^{21}$.

\section{Virus papiloma humano}

La incidencia y el número total de pacientes con CCEOF está aumentando exponencialmente ${ }^{2}$ y el aumento de CCEOF relacionado con VPH ha causado un significativo cambio en la población que recibe tratamiento ${ }^{20}$. En general, el cáncer de cabeza y cuello relacionado con el VPH tiene un pronóstico favorable, pero la falla local también ocurre y el número de pacientes considerados para cirugía de rescate de la orofaringe va a aumentar con el tiempo, en concordancia con el aumento exponencial proyectado en el número de casos de CCEOF. En una reciente serie publicada de 108 pacientes de dos grandes instituciones norteamericanas que detallan resultados de la cirugía de rescate para CCEOF, en el cual $74 \%$ fueron VPH positivos ${ }^{22}$. En general, para motivos de la presente revisión, al hablar de cánceres orofaríngeos asociados a VPH, se usa el marcador p16 como sustituto (surrogate) de verdadera asociación con VPH. Métodos más específicos de detección de VPH son mediante técnicas de PCR o de ISH (hibridación in situ), pero se acepta como parte del estudio y manejo en que casos que cumplan con un perfil de asociación a VPH el p16 es un marcador sustituto suficiente para aceptar verdadera asociación.

\section{Factores pronósticos}

En la literatura se describe una variedad de factores asociados con malos resultados de supervivencia. La etapa tumoral en el momento de la recurrencia, ha sido asociado con peores resultados después de la cirugía de rescate. Estadios III-IV, T3-4 0 N2-3 se han asociado a peores resultados de supervivencia ${ }^{23-28}$. Además, márgenes positivos en la cirugía de rescate se han correlacionado con peores resultados ${ }^{28-30}$. Enfermedad agresiva caracterizada por invasión linfovascular, recurrencias regionales concurrentes, alta graduación histológica, y afectación de la base de la lengua también se han descrito como factores con tasas de supervivencia más desfavorables $25,26,29,31$.

La presencia de p16 como marcador sustituto (surrogate) de VPH se ha asociado con resultados contradictorios de supervivencia ${ }^{20}$. Patel y cols no encontraron diferencias significativas en supervivencia después de la cirugía de rescate entre pacientes con VPH positivo y pacientes con VPH negativo ${ }^{29}$. La hipótesis de los autores es que los resultados de supervivencia similares pueden ser el resultado de una falla primaria al tratamiento con radioterapia, representando un fenotipo p16 positivo más agresivo. Estos resultados son respaldados por Sweeny y cols, quienes encontraron que los pacientes en los que la radioterapia primaria había fallado tuvieron resultados de supervivencia similares, independientemente del estado del $\mathrm{VPH}^{32}$. Patel y cols no encontraron diferencias 
estadísticamente significativas entre las tasas de recurrencia local para pacientes con p16 positivo y negativo. Sin embargo, reportaron una diferencia estadísticamente significativa respecto al número de pacientes con 16 positivo que presentaron metástasis a distancia, en comparación con pacientes p16 negativos. Además, en casos de neoplasias p16 positivas que recurrieron, todos tuvieron fracaso del tratamiento localmente 0 en conjunto con metástasis regionales $\mathrm{y} / 0$ distantes, mientras que el grupo p16 negativo tuvo fracaso del tratamiento local y/o regionalmente sin metástasis a distancia. La tasa de supervivencia sin recurrencia a cinco años para pacientes p16 positivo fue del $21 \%$, en comparación con $12 \%$ para pacientes con p16 negativo ${ }^{29}$.

El hallazgo de recurrencias distantes en pacientes con p16 positivo también se reporta en el estudio Huang y $\operatorname{cols}^{33}$, que mostró que es más probable que los pacientes con VPH positivo presentaran diseminación a múltiples órganos y sitios inusuales como pulmón, cerebro, músculo esquelético, ganglios linfáticos pericárdicos e intraabdominales. Además, la recurrencia distante se presentaba tras dos años después del tratamiento curativo, en comparación con los pacientes con p16 negativo, en quienes la recurrencia ocurre típicamente dentro de los dos primeros años tras el tratamiento. Por lo anteriormente expuesto, pareciera que el estado p16 afecta el momento y el tipo de recurrencias en pacientes con CCEOF después del tratamiento primario ${ }^{34}$. Considerando todo lo expuesto previamente, aún no es posible obtener alguna conclusión definitiva respecto a la asociación del estado de p16 con recurrencia y resultados de supervivencia. Se requiere mayor investigación para establecer la asociación entre el estado del VPH, patrones de recurrencia y resultados de supervivencia después de la cirugía de rescate.

\section{Cirugía de rescate en CCEOF vs CEL}

La evidencia disponible de artículos sobre cirugía de rescate en este tipo de condiciones corresponde principalmente a reportes de instituciones únicas, no multicéntricos ${ }^{15}$. Es destacable la diferencia en el uso de cirugía de rescate entre casos de carcinomas escamosos recurrentes orofaríngeos y laríngeos. Un estudio de 109 casos de LSCC (carcinoma escamoso laríngeo) primarios reportó cirugía de rescate en el $89 \%(39 / 44)$ de las recurrencias ${ }^{35}$. La cirugía de rescate también se realizó en $88,5 \%$ (23/26) de recurrencias en una serie de tumores de glotis ${ }^{36}$. Hay algunas series de CCEOF más grandes que reflejan la creciente incidencia de esta enfermedad. En una serie de 170 pacientes con CCEOF, 31,4\% (11/35) de las recurrencias recibió cirugía de rescate ${ }^{19}$. Una serie más grande que revisa 1.681 pacientes con CCEOF tenían 199 casos con recurrencia local, de los cuales $41(20,6 \%)$ recibió cirugía de rescate ${ }^{14}$.

Una razón potencial para que los CCEOF reciban menos cirugía de rescate con intención curativa se debe a que la enfermedad recurrente puede ser difícil de detectar clínicamente en esta zona $^{37}$. La orofaringe es más difícil de examinar, la recurrencia puede ser profunda en el tejido y puede no estar asociada con síntomas significativos hasta que se presente un estadio T avanzado. Sin embargo, actualmente hay mayor disponibilidad y uso regular de estudios con tomografía computarizada con contraste con mejor resolución, así como, mediante PET-CT, lo que permite que las recurrencias CCEOF se identifiquen de manera más anticipa$\mathrm{da}^{38,39}$. En un estudio con pacientes incluidos entre 1998 y $2005,76,2 \%$ de las recurrencias de casos de CCEOF correspondían a tumores T3 o T4 al momento de ser diagnosticados ${ }^{14}$. El estudio mediante PET-CT de la recurrencia en orofaringe permite una buena evaluación ${ }^{40}$ y proporciona datos que permiten optimizar la planificación de la resección. Además, la evaluación con PET-CT a las 12 semanas posterior a tratamiento con radioquimioterapia en persistencia de adenopatías cervicales es el estándar para estratificar la posibilidad que la masa cervical sea cáncer regional residual. Sin embargo, anteriormente, la menor disponibilidad de PET-CT para este objetivo puede haber influido en que menos pacientes puedan haber recibido cirugía de rescate.

\section{Factores de mal pronóstico}

El período libre de recurrencia que sigue al tratamiento primario de CCEOF, se ha reportado como un factor significativo en la supervivencia. Kim y cols reportaron que un período libre de 
recurrencia de seis meses o más, se asoció con mejores resultados de supervivencia a un año después de la cirugía de rescate ${ }^{27}$. Además, Zafereo y cols encontraron que el $92 \%$ de los pacientes sin intervalos libres de enfermedad, desarrollaron recurrencias con tasas de supervivencia total de menos del $20 \%$ y que, $66,7 \%$ de estos pacientes desarrollaron una segunda recurrencia dentro de los ocho meses de la cirugía de rescate ${ }^{14}$. Estos resultados indican la necesidad de un seguimiento cercano en los primeros 12 meses después de la cirugía, con estudio de reetapificación en caso de ser detectadas nuevas recurrencias. El seguimiento tanto para casos primarios como para recidivas tratadas se debe hacer de acuerdo al mismo estándar de cuidado con técnicas de imagen convencional, reservándose el PET-CT para indicaciones seleccionadas ${ }^{40}$.

La asociación entre recurrencias en estadio $T$ más avanzado y resultados quirúrgicos de rescate menos favorables se reportó en un estudio retrospectivo de 175 pacientes $^{23}$. Zafereo y cols también mostraron que un estadio T más avanzado tuvo una asociación importante con un peor resultado ${ }^{14}$. La capacidad de lograr una resección con márgenes negativos en tumores de la orofaringe puede ser técnicamente más difícil, en consecuencia, esto puede haber representado un obstáculo para la cirugía de rescate en el pasado. El tratamiento quirúrgico usualmente incluiría un abordaje mediante un swing mandibular con mandibulotomía o una resección compuesta con mandibulectomía y reconstrucción mediante colgajo libre. En un estudio de 29 pacientes sometidos a cirugía de rescate, se logró un margen negativo en 17 pacientes $(58,6 \%)^{28}$. Resultados similares se observaron en un estudio de Toronto, en el que se lograron márgenes negativos en 12 pacientes $(35,3 \%)$ de la serie, 16 pacientes $(47,1 \%)$ obtuvieron márgenes cercanos $(<5 \mathrm{~mm})$ y 6 pacientes $(17,6 \%)$ tenían un margen positivo ${ }^{29}$. Hay y cols publicaron sus resultados de cirugía de rescate en casos recurrente de LSCC y CCEOF, en los cuales lograron márgenes de resección negativo después de la cirugía de rescate en 44/61 pacientes $(72,1 \%)^{21}$. No presentaron diferencias significativas para lograr márgenes negativos entre ambas zonas, obteniendo márgenes negativos en $63,9 \%$ de los pacientes con tumores laríngeos y $84 \%$ de pacientes con tumores orofaríngeos. Describieron, que la mayor disponibilidad de reconstrucción mediante colgajo libre en los casos más recientes, permitió al equipo ablativo mayor confianza para realizar la resección y en consecuencia esto puede haber repercutido en su buena tasa de márgenes negativos. En este artículo la obtención de un margen de resección negativo también fue asociado con una mejor supervivencia general, con un promedio de 38 meses en pacientes en los que se logró márgenes de resección negativa, en comparación con una media de 9 meses en pacientes con márgenes positivos ${ }^{21}$. Se ha descrito ampliamente en la literatura que los sitios no laríngeos tienen peores resultados de supervivencia tras cirugía de rescate, sin embargo, en el estudio de Hay y cols el sitio del tumor no significó una diferencia estadísticamente significativa en la tasa de supervivencia, en donde la mediana de supervivencia de LSCC y CCEOF fue de 23 y 26 meses, respectivamente ${ }^{21}$.

\section{Supervivencia}

En una serie de Toronto de 34 pacientes tratados con cirugía de rescate para CCEOF recurrente, la supervivencia a los 2, 3 y 5 años fue del $62 \%$, $41 \%$ y $25 \%{ }^{29}$. Reportaron que no se encontraron diferencias en resultados entre tumores con VPH positivo y negativo, pero también se destaca en el artículo la importancia de obtener márgenes negativos para la supervivencia. En una revisión de CCEOF recurrente en el Hospital John Hopkins en Baltimore se estudiaron 108 tumores recurrentes y reportaron que tanto los tumores con VPH positivo y negativo se beneficiaron de cirugía de rescate ${ }^{22}$. A pesar de la baja supervivencia a largo plazo de la cirugía de rescate en estos casos, la cirugía parece ser la mejor oportunidad para pacientes con enfermedad recurrente. Las tasas de supervivencia de pacientes de cirugía de rescate fueron significativamente más altas en comparación con las de pacientes tratados con quimioterapia paliativa, reirradiación o cuidados paliativos, con tasas de supervivencia global a 3 años de $32 \%, 4 \%$ y $5 \%$ respectivamente ${ }^{14}$. Röösli y col. describieron una tasa de supervivencia a cinco años de cirugía de rescate tras radioterapia primaria de $25 \%$, en comparación con $40 \%$ de cirugía primaria seguida 
de radioterapia ${ }^{41}$. Patel y cols reportaron que la tasa de supervivencia de pacientes que no recibieron cirugía de rescate era $19 \%, 9 \%$ y $2 \%$ a dos, tres y cinco años, respectivamente ${ }^{29}$.

En una revisión de 18 estudios, 17 series de casos y 1 estudio de caso-control que incluyó a pacientes sometidos a cirugía de rescate para CCEOF recurrente, la supervivencia general para 2 y 5 años fue de $52 \%$ y $30 \%$ respectivamente. Los estudios realizados antes del año 1990 presentaban una tasa de supervivencia a dos y cinco años de 30,3\% y $24,2 \%$, mientras que los estudios realizados después de 1990 presentaban tasas de supervivencia de $64,9 \%$ y $32,6 \%$ respectivamente ${ }^{42}$. La selección de pacientes es primordial para lograr obtener mejores resultados de supervivencia en este grupo de pacientes. Entre los factores favorables en contexto de cirugía de rescate CCEOF identificados en esta revisión se encuentran: estadio T1-2, ausencia de metástasis regionales y a distancia, estadios I 0 II, estado según la Sociedad Americana de Anestesiólogos (ASA) 1 ó 2, no bebedores 0 aquellos que hayan presentado un intervalo libre de enfermedad de seis meses 0 más ${ }^{42}$.

\section{Selección apropiada de pacientes para cirugía de rescate}

Se debiese tener precaución al decidir realizar una cirugía de rescate en pacientes con factores desfavorables. La selección adecuada de pacientes para la cirugía de rescate es crucial dada la morbilidad y mortalidad que involucra. Por lo tanto, es importante identificar y valorar los factores que puedan predecir los resultados después de la cirugía de rescate. Zafereo y cols concluyeron que los candidatos favorables para cirugía de rescate son pacientes jóvenes, con intervalos libres de enfermedad después del tratamiento primario e idealmente recurrencias pequeñas en los cuales sea posible obtener márgenes negativos y ausencia de recurrencia en el cuello ${ }^{14}$. La definición utilizada de intervalo libre de enfermedad fue de una respuesta completa demostrable del tumor inicial al tratamiento con posterior desarrollo de un segundo CCEOF, después de un intervalo libre de enfermedad de seis semanas ${ }^{14}$. En contraste, se ha informado que presentan menor supervivencia tras cirugía de rescate pacientes con mal estado de salud general y según lo identificado por el índice de comorbilidad de edad de Charlson o de la clasificación de estado $\mathrm{ASA}^{23-26}$. Es recomendable realizar una discusión y valoración de estos factores para la determinación de cirugía de rescate y al momento de aconsejar al paciente y familiares.

\section{Complicaciones}

Las complicaciones posoperatorias después de una cirugía de rescate se reportan entre $17 \%$ a $78 \%$ de los pacientes, y éstas pueden generar morbilidad y mortalidad significativas. Pueden presentarse desde infecciones de heridas leves hasta rupturas de arteria carótida ${ }^{18-22,24,25,28,32,33}$. La literatura actual ha demostrado que la cirugía de rescate se asocia con más complicaciones posoperatorias al compararlos con cirugía primaria, particularmente después de quimiorradioterapia ${ }^{39}$. De la misma manera, dosis altas de radioterapia más de 60 Gy corresponde a un factor importante en las tasas de falla de colgajo locorregional o colgajo libre. Basso Ricci y cols reportaron una mayor incidencia de fístulas cuando la cirugía de rescate se realizó junto a disecciones de cuello ${ }^{43}$. También se ha reportado una estrecha relación entre pacientes con comorbilidades tales como: diabetes mellitus, antecedentes de infarto agudo al miocardio, enfermedad vascular periférica y enfermedad pulmonar con complicaciones locales y generales ${ }^{27}$.

\section{CONCLUSIÓN}

Este artículo corresponde a una revisión del estado actual de las tasas de supervivencia de pacientes con carcinoma escamoso de orofaringe tratados mediante cirugía de rescate. Se han descrito también los hallazgos actuales respecto a los patrones de recurrencia, factores pronósticos y relación con virus papiloma humano. Parece haber una mejoría en la tasa de supervivencia general en los últimos 30 años, posiblemente debido a una asociación con el estado de VPH y la mayor disponibilidad de reconstrucción mediante colgajos libres que permiten una resección amplia con mayor tasa de obtención de márgenes negativos.

En la actualidad el aumento del cáncer de orofaringe y su cambio epidemiológico ofrece una 
nueva perspectiva, en el cual ya se han reportado tasas de supervivencia general similar en comparación con el carcinoma escamoso recurrente de laringe. Los estudios de CCEOF debiesen integrar un informe estandarizado del estado de VPH en Ios resultados para permitir y favorecer un análisis

\section{BIBLIOGRAFÍA}

1. Ferlay J, Shin hr, Bray F, Forman D, Mathers C, PARKIN DM. GLOBOCAN 2008 v2.0, Cancer Incidence and Mortality Worldwide: IARC CancerBase No. 10. Lyon: International Agency for Research on Cancer, 2010.

2. Chaturvedi AK, Engels EA, Pfeiffer RM, Hernandez BY, XıAO W, KIm E et al. Human papillomavirus and rising oropharyngeal cancer incidence in the United States. J Clin Oncol2011; 29: 4294301.

3. Hong aM, Grulich AE, Jones D, Lee CS, Garland SM, DobiIns TA et al. Squamous cell carcinoma of the oropharynx in Australian males induced by human papillomavirus vaccine targets. Vaccine 2010; 28: 3269-72.

4. GILLIson ML, Lowy DR. A causal role for human papillomavirus in head and neck cancer. Lancet 2004; 363: 1488-9.

5. Liu R, Gullane P, Brown D, Irish J. Pectoralis major myocutaneous pedicled flap in head and neck reconstruction: retrospective review of indications and results in 244 consecutive cases at the Toronto General Hospital. J Otolaryngol 2001; 30: 34-40.

6. WarnakUlasuriya S. Global epidemiology of oral and oropharyngeal cancer. Oral Oncol2009; 45: 309-16.

7. Jesse RH, Sugarbaker EV. Squamous cell carcinoma of the oropharynx: why we fail. $A m \mathrm{~J}$ Surg 1976; 132: 435-8.

8. Chen AY, Fedewa S, Zhu J. Temporal trends in the treatment of early- and advanced-stage laryngeal cancer in the United States, 1985-2007. Arch Otolaryngol Head Neck Surg 2011; 137: 101724.

9. Maddox PT, Davies L. Trends in total laryngectomy in the era of organ preservation: a populationbased study. Otolaryngol Head Neck Surg 2012; 147: 85-90. estadístico respecto a este factor. En esta revisión se describen varios factores identificados como factores asociados a la tasa de supervivencia, que esperamos ayude a los cirujanos tratantes en la selección y manejo de sus pacientes en escenario de cirugía de rescate.

10. Chen aY, Schrag N, Hao Y, Stewart A, Ward E. Changes in treatment of advanced oropharyngeal cancer, 1985-2001. Laryngoscope 2007; 117: 16-21.

11. Chen AY, Zhu J, Fedewa S. Temporal trends in oropharyngeal cancer treatment and survival: 1998-2009. Laryngoscope 2014; 124: 131-8.

12. Lyhne NM, Johansen J, Kristensen CA, Andersen E, Primdahl H, Andersen LJ et al. Pattern of failure in 5001 patients treated for glottic squamous cell carcinoma with curative intent-a population based study from the DAHANCA group. Radiother Oncol2016; 118: 257-66.

13. Bird T, De Felice F, Michaelidou A, Thavaraj $S$, Jeannon JP, Lyons A et Al. Outcomes of intensitymodulated radiotherapy as primary treatment for oropharyngeal squamous cell carcinoma-a European single institution analysis. Clin Otolaryngol 2017; 42: 115-22.

14. Zafereo ME, Hanasono MM, Rosenthal DI, Sturgis EM, Lewin JS, Roberts DB et aL. The role of salvage surgery in patients with recurrent squamous cell carcinoma of the oropharynx. Cancer 2009; 115: 5723-33.

15. Ho AS, Kraus DH, Ganly I, Lee NY, Shah JP, Morris LG. Decision making in the management of recurrent head and neck cancer. Head Neck2014; 36: $144-51$.

16. GoodWINWJ JR. Salvage surgery for patients with recurrent squamous cell carcinoma of the upper aerodigestive tract: when do the ends justify the means? Laryngoscope 2000; 110: 1-18.

17. Kowalski LP, Carvalho AL. Natural history of untreated head and neck cancer. Eur J Cancer 2000; 36: 1032-37.

18. Ganly I, Patel S, Matsuo J, Singh B, Kraus D, Boyle $J$ Et AL. Postoperative complications of salvage total laryngectomy. Cancer 2005; 103: 2073-81.

19. Kano S, Homma A, Hayashi R, Kawabata K, Yoshino K, IWAE $S$ et AL. Salvage surgery for recurrent 
oropharyngeal cáncer after chemoradiotherapy. Int J Clin Oncol 2013; 18: 817-23.

20. Ang KK, Harris J, Wheeler R, Weber R, Rosenthal DI, NguYen- Tan PF et AL. Human papillomavirus and survival of patients with oropharyngeal cancer. N Engl J Med 2010; 363: 24-35.

21. Hay A, Simo R, Hall G. et al. Outcomes of salvage surgery for the oropharynx and larynx: a contemporary experience in a UK Cancer Centre. Eur Arch Otorhinolaryngol 2019; 276 : 1153-59.

22. Guo T, Qualliotine JR, Ha PK, Califano JA, Kim Y, SAunders JR Et AL. Surgical salvage improves overall survival for patients with HPV-positive and HPV-negative recurrent locoregional and distant metastatic oropharyngeal cancer. Cancer 2015; 121: 1977-84.

23. Bachar GY, Goh C, Goldstein DP, O'Sullivan B, Irish JC. Longterm outcome analysis after surgical salvage for recurrent tonsil carcinoma following radical radiotherapy. Eur Arch Otorhinolaryngol 2010; 267: 295-301.

24. Culié D, Benezery K, Chamorey E, Ettaiche M, Fernandez J, Poissonnet G et al. Salvage surgery for recurrent oropharyngeal cancer: postoperative oncologic and functional outcomes. Acta Otolaryngol 2015; 135: 1323-9.

25. Gehanno P, Depondt J, Guedon C, Keballi C, Koka V. Primary and salvage surgery for cancer of the tonsillar region: a retrospective study of 120 patients. Head Neck 1993; 15: 185-9.

26. Kásler M, Fodor J, Oberna F, Major T, Polgár C, TAKÁCSI-NAGY Z. Salvage surgery for locoregional failure after definitive radiotherapy for base of tongue cancer. In Vivo 2008; 22: 803-6.

27. Kim J, Kim S, Albergotti WG, Cho PA, Kaplan DJ, АввеRBock $S$ et al. Selection of ideal candidates for surgical salvage of head and neck squamous cell carcinoma: effect of the Charlson AgeComorbidity Index and oncologic characteristics on 1-year survival and hospital course. JAMA Otolaryngol Head Neck Surg 2015; 141: 105965.

28. Nichols AC, Kneuertz PJ, Deschler DG, Lin DT, Emerick KS, Clark JR et al. Surgical salvage of the oropharynx after failure of organ-sparing therapy. Head Neck 2011; 33: 516-24.

29. Patel SN, Cohen MA, Givi B, Dixon BJ, Gilbert RW, Gullane PJ et Al. Salvage surgery for locally recurrent oropharyngeal cancer. Head Neck 2016; 38: E658-64.

30. White H, Ford S, Bush B, Holsinger FC, Moore E, GHANem T ET AL. Salvage surgery for recurrent cancers of the oropharynx comparing TORS with standard open surgical approaches. JAMA Otolaryngol Head Neck Surg 2013; 139: 773-8.

31. Omura G, Saito Y, Ando M, Kobayashi K, Ebihara Y, Yamasoba T et AL. Salvage surgery for local residual or recurrent pharyngeal cancer after radiotherapy or chemoradiotherapy. Laryngoscope 2014; 124 : 2075-80.

32. Sweeny L, Rosenthal EL, Clemons L, Stevens tM, Cook Mclntosh ER, Carroll WR. Outcomes after surgical salvage for recurrent oropharyngeal squamous cell carcinoma. Oral Oncol 2016; 60: 118-24.

33. Huang SH, Perez-Ordonez B, Weinreb I, Hope A, Massey C, Waldron JN et al. Natural course of distant metastases following radiotherapy or chemoradiotherapy in HPV-related oropharyngeal cancer. Oral Oncol 2013; 49: 79-85.

34. Fakhry C, Zhang Q, Nguyen-Tan PF, Rosenthal D, ElNaggar A, Garden AS et al. Human papillomavirus and overall survival after progression of oropharyngeal squamous cell carcinoma. J Clin Oncol 2014; 32: 3365-73.

35. Stoeckli SJ, Pawlik AB, Lipp M, Huber A, Schmid $S$. Salvage surgery after failure of nonsurgical therapy for carcinoma of the larynx and hypopharynx. Arch Otolaryngol Head Neck Surg 2000; 126: 1473-7.

36. Mclaughlin MP, Parsons JT, Fein DA, et al. Salvage surgery after radiotherapy failure in T1-T2 squamous cell carcinoma of the glottic larynx. Head Neck 1996; 18: 229-35.

37. Eckardt A, Barth El, Kokemueller H, Wegener $G$. Recurrent carcinoma of the head and neck: treatment strategies and survival analysis in a 20-year period. Oral Oncol 2004; 40: 427-32.

38. Frakes JM, Strom T. Detection of recurrence in HPV associated oropharynx squamous cell carcinoma. In: Presented at: 2016 multidisciplinary head and neck cancer symposium; February 18-20, 2016; Scottsdale, AZ. Abstract 62016.

39. Yao M, Smith RB, Hoffman HT, Funk GF, Lu M, Menda Y ET AL. Clinical significance of postradiotherapy [18F]-fluorodeoxyglucose positron emission tomography imaging in management of head- 
and-neck cancer-a long-term outcome report. Int J Radiat Oncol Biol Phys 2009; 74: 9-14.

40. Bird T, Barrington S, Thavaraj S, Jeannon JP, Lyons A, OAKLEY R Et Al. (18)F-FDG PET/CT to assess response and guide risk-stratified follow-up after chemoradiotherapy for oropharyngeal squamous cell carcinoma. Eur J Nucl Med Mol Imaging 2016; 43: 1239-47.

41. Röösli C, Studer G, Stoeckli SJ. Salvage treatment for recurrent oropharyngeal squamous cell carcinoma. Head Neck 2010; 32: 989-96.

42. KaO SS, Ool EH. Survival outcomes following salvage surgery for oropharyngeal squamous cell carcinoma: systematic review. J Laryngol Otol 2018; 132: 299-313.

43. Basso Ricci S, Grandi C, Salvatori P. On the problem of failures of radiotherapy in the treatment of oropharyngeal carcinoma; salvage surgery. Rays 1986; 11: 127-31. 